Cuckow, F. W. \& Klieneberger-Nobel, E. (1955). J. gen. Microbiol. 13, 149-154.

\title{
Further Studies of Organisms of the Pleuropneumonia Group by Electron Microscopy
}

\author{
By F. W. CUCKOW \\ Chester Beatty Research Institute, Institute of Cancer Research, \\ Royal Cancer Hospital, London \\ AND E. KLIENEBERGER-NOBEL \\ The Lister Institute of Preventive Medicine, London
}

\begin{abstract}
SUMMARY: The growth on solid medium of organisms of the pleuropneumonia group as seen in electron micrographs was compared with the growth in liquid media described in a previous paper (Klieneberger-Nobel \& Cuckow, 1955). There is no evidence of a rigid cell wall in these organisms, a finding consistent with those electron micrographs of growth in liquid media and in light microscopical preparations. The newly formed minimal reproductive units, hitherto never demonstrated in the light microscope except when they have grown to a larger size, are here demonstrated in large numbers as a uniform phase of small, delicate elements, the diameter of which measures $c .100 \mathrm{~m} \mu$. in the electron micrographs.
\end{abstract}

In a previous paper (Klieneberger-Nobel \& Cuckow, 1955) we demonstrated the electron micrographic appearances of some organisms of the pleuropneumonia group grown in liquid media. The present study is mainly concerned with the appearance of these organisms when grown on solid media.

\section{MATERIALS AND METHODS}

The strains used were: the organisms of agalactia and of pleuropneumonia of goats of our previous study: an organism of pleuropneumonia of cattle sent from Africa; an organism designated 'Cheng' (Klieneberger-Nobel \& Cheng; to be published) isolated from a lung abscess of a bronchiectatic rat; an organism from sewage isolated by Laidlaw \& Elford (1936), designated 'Laidlaw A'.

The methods employed with organisms grown in liquid medium were slightly altered to suit growth on solid medium. A 'formvar' solution in chloroform was poured over a slice of appropriate agar medium resting on a sterile slide, thus producing a membrane on its surface. The organisms were freshly grown in liquid medium, spun down and washed once in TyrodeRinger solution. The sediment was resuspended in Tyrode-Ringer solution and diluted to an opacity which varied with the strain, but which was usually just appreciable by the naked eye. With a fine capillary pipette droplets of this suspension were placed on the formvar membrane. Each preparation was incubated in a Petri dish, which for long incubation was kept in a moist chamber. The water of the droplet gradually evaporated leaving a round, distinct, sometimes slightly opaque area. Drying was sometimes speeded up by a pre-drying of the membrane-covered agar-slices in the incubator. After 
the desired period of incubation the formvar membranes were floated on Tyrode-Ringer solution for 15-30 min., then floated on $40 \%$ formalin, for $3 \mathrm{~min}$., and then on distilled water for $1 \mathrm{hr}$. The parts of the membranes which carried the droplets were placed on grids, dried, shadowed and photographed under the electron microscope at $60 \mathrm{kV}$. Uninoculated control preparations showed nothing significant.

\section{Description of the electron micrographs}

Plate 1, fig. 1, shows the 'Laidlaw A' organism grown for $18 \mathrm{hr}$. at room temperature. Some of the resulting delicately grown patches contain roundish opaque bodies which are similar to those often seen in liquid media. However, they differ in that their edges are thinner, more irregular and hazy, as though they had begun to spread. The edges of adjacent forms are connected with each other so that the boundaries of single organisms are no longer distinguishable in some of the grape-like agglomerations.

Plate 1, fig. 2, shows the organism of pleuropneumonia of cattle grown for $18 \mathrm{hr}$. at room temperature. The growth is only scanty and delicate. Some of the organisms are no larger than those from liquid media, but their edges are less definite. Others appear to have enlarged without subdividing, and to have become thinner and more transparent to the electron beam. The larger bodies vary in transparency.

Plate 1, fig. 3, shows the goat pleuropneumonia organism, a quickly growing strain. After $4 \frac{1}{2} \mathrm{hr}$. at $37^{\circ}$ there was not a single organism which resembled the forms found in growths from serum broth. All the organisms here are spread out on the membrane and are extremely thin; the organisms at the edge of the colony are particularly thin in their outermost parts. This appearance is common in stained preparations of growths on solid medium and is also observed in living specimens under the 'ultropak' microscope. The area in the print shown has a superficial resemblance to a tissue section, an appearance often seen in stained and in phase-contrast preparations of colonies of pleuropneumonia-like organisms.

Plate 1, fig. 4, shows the edge of a colony of the sewage organism 'Laidlaw A'. This organism grows even more rapidly than the goat pleuropneumonia organism. The culture was grown on a pre-dried membrane for $5 \mathrm{hr}$. at $37^{\circ}$ and then $18 \mathrm{hr}$. at room temperature in order to obtain a more advanced growth than in the specimens so far described. A group of fairly large forms is seen surrounded by minimal reproductive units.

Plate 1, fig. 5, shows growth from a 2-day serum broth culture of the organism of agalactia. This picture is included to show that discrete minimal reproductive units occur in liquid media, a fact which we failed to illustrate satisfactorily in previous work. The minimal reproductive units seen here are of the same size as those grown on the solid medium. All these units are fairly uniform and have a diameter of $c .100 \mathrm{~m} \mu$. in the electron micrographs.

Plate 1, fig. 6, shows the strain 'Cheng' grown for $18 \mathrm{hr}$. at $37^{\circ}$. A large number of minimal reproductive units, singly and in small groups, are 
scattered over the field. There are two larger forms with irregular edges which may represent 'mother' forms from which the minimal reproductive forms develop.

Plate 2, fig. 7, shows the sewage organism 'Laidlaw A'. There are minimal reproductive units as well as some very large flat forms, of a kind which frequently occur on the surface of solid media.

Plate 2, fig. 8, shows the sewage organism 'Laidlaw A'; the appearances are consistent with the production of minimal reproductive units by sprouting.

Plate 2, fig. 9, shows a micro-colony of the 'Laidlaw A' organism. In various places round the edge the large organisms seem to have been replaced by collections of minimal reproductive units lying in a continuous matrix. In a few of the large organisms granules of about the size of the minimal reproductive units can be seen.

Plate 2, fig. 10, shows some of the organisms of agalactia from solid medium with a peripheral concentration of material, a feature so characteristic for the much smaller organisms which occur in liquid media. These thickenings appear particularly when cultivation on solid medium takes place in a moist atmosphere. In stained preparations these peripheral thickenings may easily be interpreted, and often have been so interpreted, as cell walls. However, in the electron micrograph shown here it seems to be only a concentration of material, often unevenly distributed. It may indeed be material from which minimal reproductive units are produced.

\section{DISCUSSION}

The variety of electron micrographic appearances among pleuropneumonialike organisms grown in liquid media and organisms grown on solid media is great. In liquid media small spheres, oval bodies, disks and filaments occur exclusively; on solid media the forms have little depth, but are spread widely over the membrane in thin sheets or in leaf-like and sometimes amoeboid forms. This diversity, dependent on external conditions, is evident in the light microscope, but the electron micrographic evidence is even more striking. From consideration of stained and darkfield preparations and the results of filtration analysis, most workers agree that the organisms of the pleuropneumonia group lack a definite cell wall, and that they produce during their growth small corpuscles, the minimal reproductive units, which can pass fine filters and reproduce.

The electron micrographs of specimens from liquid and solid media reveal no rigid cell walls. Moreover, the thin hazy edge of the forms as seen in many of the electron micrographs here (for example, Pl. 1, fig. 1), the apparent lack of boundaries between neighbouring forms (Pl. 1, fig. 3, and Pl. 2, fig. 9), and the occurrence of material links between neighbouring forms (Pl. 2, fig. 10) bear out the view of the non-existence of a rigid cell wall.

However, one particular appearance of forms in stained preparations has sometimes been misinterpreted. A ring of darkly staining material may appear at the outer boundary of various forms. In older specimens darkly staining 
'granules' appear within this ring which, as noted above, has been taken for a cell wall. From the electron micrographs (e.g. Pl. 2, fig. 10) it can be seen that an irregular rim of opaque material is often produced round some forms or round parts of some forms, which may give the impression of a cell wall in stained specimens. Yet the electron microscope shows that there is no cell wall, but a rim of concentrated material which corresponds to the material concentrated round the edges of the much smaller forms grown in serum broth, which, as shown previously (Klieneberger-Nobel \& Cuckow, 1955) break up into minimal reproductive units. The electron microscope thus provides new evidence of the absence of a rigid cell wall in the pleuropneumonia group of organisms.

Minimal reproductive units occur in liquid as well as on solid media. Elford's (1929) filtration analysis of the organisms of pleuropneumonia of cattle and agalactia of sheep and goats proved that a large number of elements in these cultures can pass through fine Gradocol membranes down to the end-point of $0.25 \mu$. average pore diameter; some even pass through slightly finer membranes. According to Elford the diameter of the small units lies between 125 and $175 \mathrm{~m} \mu$; according to our own unpublished experiments (E. K.-N.) their diameter is $100-150 \mathrm{~m} \mu$. The minimal reproductive units can be seen in Pl. 1, figs. 4-6 and Pl. 2, figs. 7-9. It appears from these pictures that they are about $100 \mathrm{~m} \mu$. in diameter and represent a phase of nearly uniform size and appearance. They may be slightly larger in the living state because the electron micrographs have been prepared from dried fixed material. Their diameters as estimated from both the electron micrographs and the filtration experiments are in good agreement.

Very few workers have demonstrated small units in stained preparations or by dark-ground microscopy. Turner (1935) showed some 'granules' in his excellent darkground pictures, and similar granules appear in ultropak and stained preparation pictures (Klieneberger \& Smiles, 1942). They are also occasionally visible in the pictures of Dienes $(1945 ;$ pl. 3 , figs. 1, 2). The granules in the photographs of Klieneberger \& Smiles were fewer in number, further apart and stand out more brilliantly than the minimal reproductive units in the electron micrographs. The latter are too small and too delicate to be visible in the light microscope, though they would be visible after an increase in size and opacity (cf. some of the units in Pl. 1, figs. 4, 5; Pl. 2, fig. 7). It follows that the 'minimal' reproductive units seen in the light microscope are no longer minimal but have grown into larger organisms.

The interpretation we offer of our electron micrographs is as follows. There is a phase of small corpuscles, the minimal reproductive units, which on the surface of solid media grow into larger flat organisms which have no rigid cell walls, but are often characterized by a soft edge. The thickness of these flat forms is of the same order as the diameter of the minimal reproductive units. The units appear to form in the substance of the flat organisms, which then degenerate to leave a single layer of discrete minimal reproductive units on the surface of the medium. In some cases the minimal reproductive units appear to arise from the end of prolongations that sprout from the edge of the 
large forms. Though the appearances of organisms grown in liquid and on solid media differ widely we consider that there is no fundamental difference of development. In the liquid medium the forms are small and produce few minimal reproductive units, while on solid medium they are large and produce a correspondingly large number of minimal reproductive units.

Electron micrographs of organisms of the pleuropneumonia group have been published by Smith, Hillier \& Mudd (1948), Freundt (1954), Dienes (1953) and Liebermeister (1953). The methods used by these authors do not appear to be the best adapted for the demonstration of these organisms under the electron microscope, because the results, though confirming what was already known from studies by light microscopical methods, did not bring to light new information in spite of the greater resolving power available. 'The electron micrographs illustrated here and in our previous paper now show, however, that the pleuropneumonia group of organisms can be studied to advantage under the electron microscope.

\section{REFERENCES}

Dienes, L. (1945). Morphology and nature of the pleuropneumonia group of organisms. J. Bact. 50, 441 .

Dienes, L. (1953). Electron micrographs made from $L$ forms of Proteus and two human strains of pleuropneumonia-like organisms. J. Bact. 66, 280.

Elford, W. J. (1929). Ultra-filtration methods and their application in bacteriological and pathological studies. Brit. J. exp. Path. 10, 126.

Freundt, E. A. (1954). Morphological and biochemical investigations of human pleuropneumonia-like organisms (Micromyces). Acta path. microbiol. scand. 34, 127.

Klieneberger-Nobex, E. \& Cuckow, F. W. (1955). A study of organisms of the pleuropneumonia group by electron microscopy. J. gen. Microbiol. 12, 95.

Kuieneberger, E. \& Smiles, J. (1942). Some observations on the developmental cycle of the organisms of bovine pleuropneumonia and related organisms. J. Hyg., Camb. 42, 110.

Laidlaw, P. P. \& Elford, W. J. (1936). A new group of filterable organisms. Proc. roy. Soc. B, 120, 292.

Liebermeister, K. (1953). Untersuchungen zur Morphologie der Pleuropneumonia(PPLO) gruppe. Z. Naturf. 86, 757.

Smith, W. E., Hillier, J. \& MUdD, S. (1948). Electron micrograph studies of two strains of pleuropneumonia-like $(\mathrm{L})$ organisms of human derivation. J. Bact. $56,589$.

Turner, A. W. (1935). A study of the morphology and life cycles of the organism of pleuropneumonia contagiosa bovum (Borrelomyces peripneumoniae nov.gen.) by observation in the living state under darkground illumination. J. Path. Bact. 41, 1 .

\section{EXPLANATION OF PLATES}

(Magnification: $\times 12,000$ throughout.)

\section{Plate 1}

Fig. 1. Sewage organisms 'Laidlaw A' grown on solid medium at room temperature for $18 \mathrm{hr}$.

Fig. 2. The organism of pleuropneumonia of cattle grown on solid medium at room temperature for $18 \mathrm{hr}$.

Fig. 3. The organism of pleuropneumonia of goats grown on solid medium at $37^{\circ}$ for $4 \frac{1}{2} \mathrm{hr}$. 
Fig. 4. Sewage organism 'Laidlaw $A$ ' grown on solid medium at $37^{\circ}$ for $5 \mathrm{hr}$. and at room temperature for an additional $18 \mathrm{hr}$.

Fig. 5. The organism of agalactia of goats grown in liquid medium at $37^{\circ}$ for 2 days.

Fig. 6. The organism causing bronchopneumonia in rats grown on solid medium at $37^{\circ}$ for $18 \mathrm{hr}$.

\section{Plate 2}

Fig. 7. Sewage organism 'Laidlaw A' grown on solid medium at $37^{\circ}$ for $5 \mathrm{hr}$. and at room temperature for an additional $18 \mathrm{hr}$.

Fig. 8. Sewage organisms 'Laidlaw $A$ ' grown on solid medium at room temperature for $18 \mathrm{hr}$.

Fig. 9. Sewage organism 'Laidlaw A' grown on solid medium at $37^{\circ}$ for $5 \mathrm{hr}$. and at room temperature for an additional $18 \mathrm{hr}$.

Fig. 10. The organism of agalactia of goats grown on solid medium in moist chamber at $37^{\circ}$ for $24 \mathrm{hr}$.

(Received 24 January 1955)

\section{ADDENDUM}

After this paper had been submitted we saw the paper of H. E. Morton, J. G. Lecce, J. J. Oskay and N. H. Coy (J. Bact. 68, 697, 1954). Their electron micrographs of pleuropneumonia-like organisms from man and chickens show appearances comparable to ours. 
Journal of General Microbiology, Vol. 13, No. 1
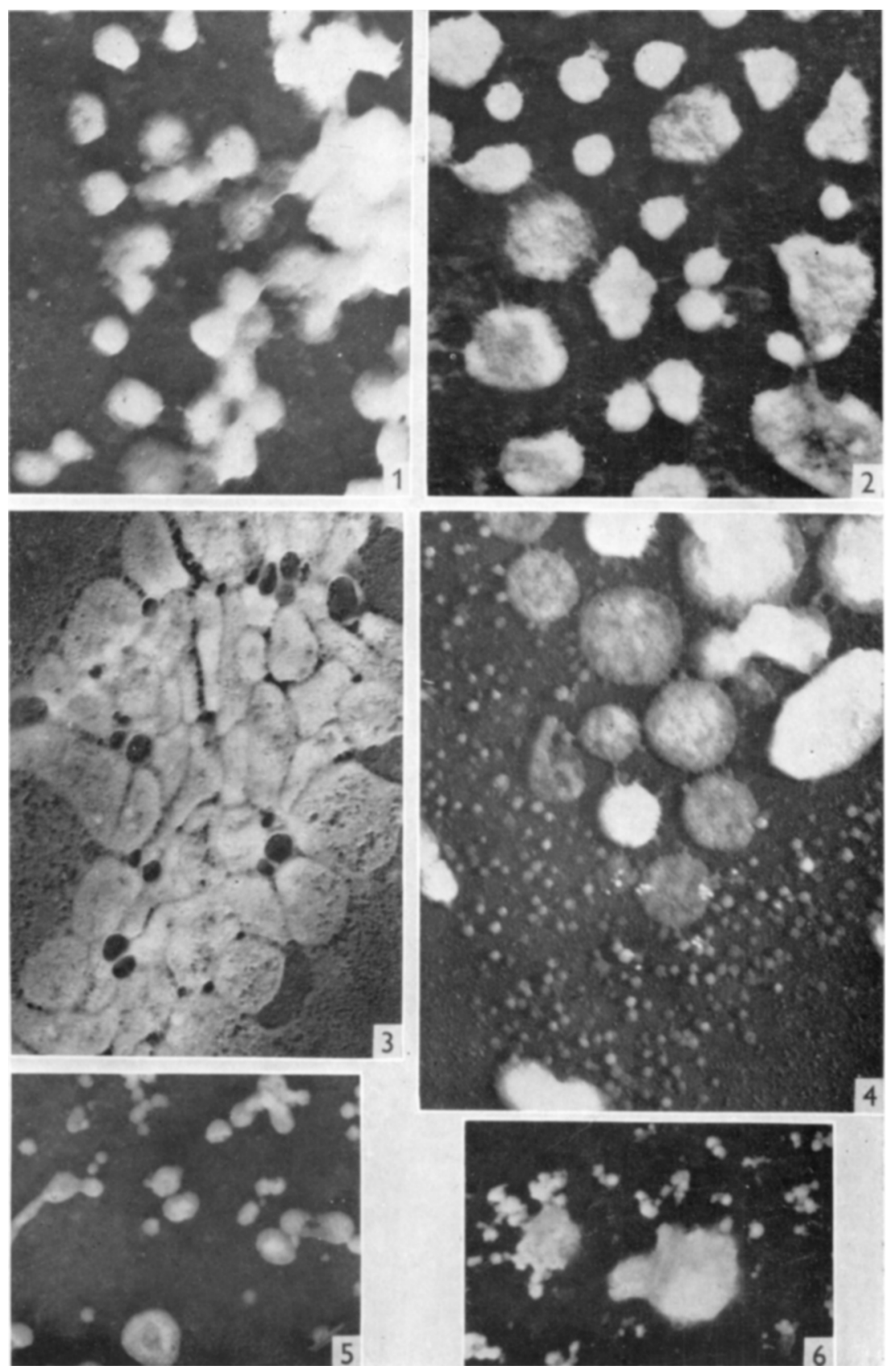

F. W. Cuckow and E. Klieneberger-Nobei,-Further studies of the pleuropneumonia group. Plate 1 
Journal of General Microbiology, Vol. 13, No. 1
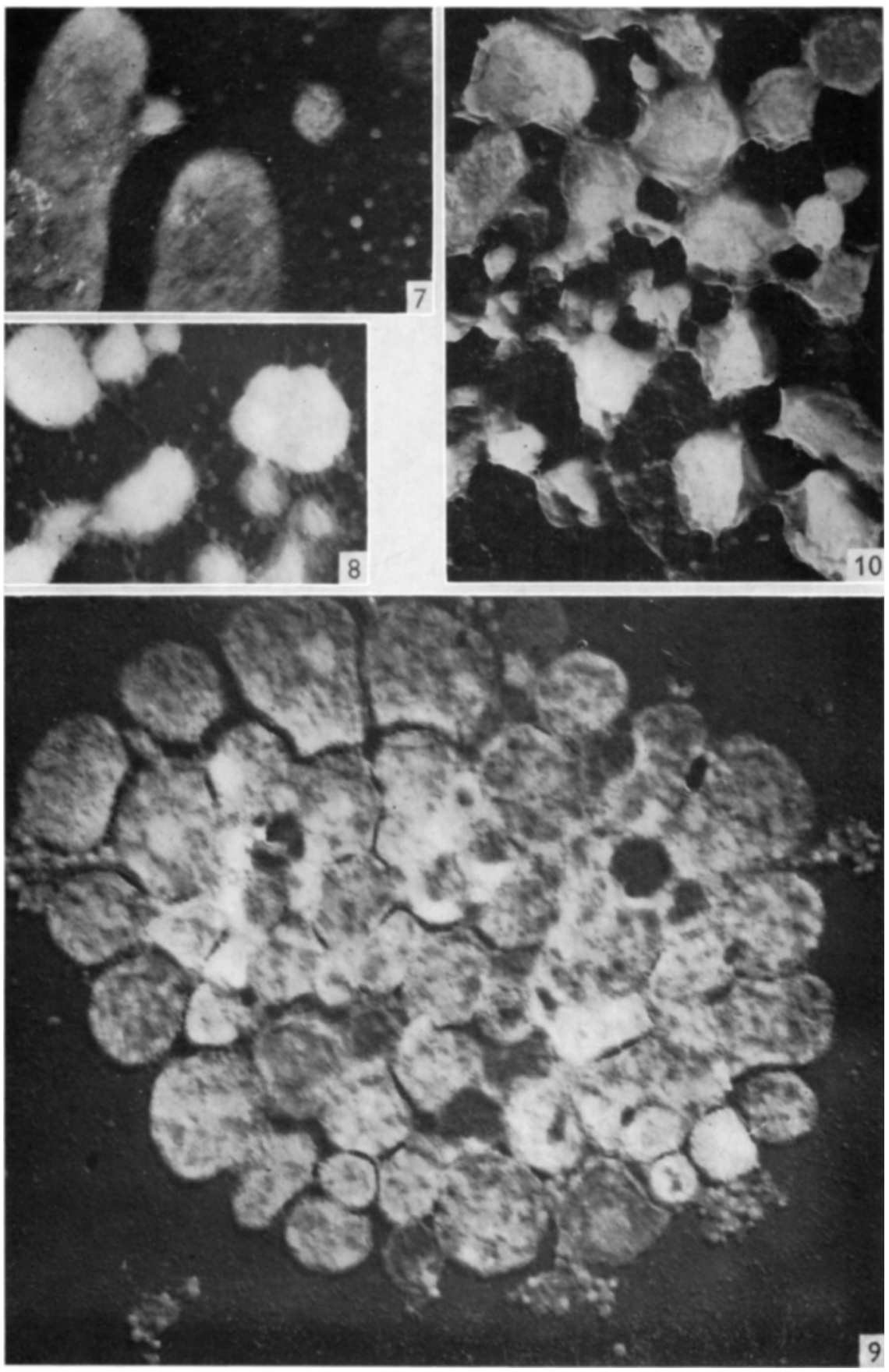

F. W. Cuckow and E. Kllieneberger-Nobei-Further studies of the pleuropneumonia group. Plate 2 